\title{
A unified approach to the important protein kinase inhibitor balanol and a proposed analogue
}

\author{
Tapan Saha, Ratnava Maitra and Shital K. Chattopadhyay ${ }^{*} \S$
}

\section{Full Research Paper}

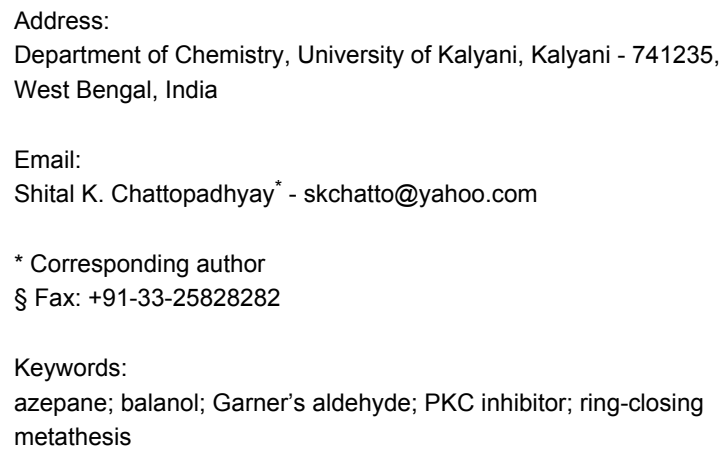

Beilstein J. Org. Chem. 2013, 9, 2910-2915. doi:10.3762/bjoc.9.327

Received: 06 August 2013

Accepted: 07 November 2013

Published: 19 December 2013

Associate Editor: S. Bräse

(C) 2013 Saha et al; licensee Beilstein-Institut. License and terms: see end of document.

\section{Abstract}

A common approach to the important protein kinase inhibitor (-)-balanol and an azepine-ring-modified balanol derivative has been developed using an efficient fragment coupling protocol which proceeded in good overall yield.

\section{Introduction}

Protein kinase C (PKC) is a family of phospholipid-dependent kinases that phosphorylate serine and threonine residues of a substrate protein by transferring a phosphate group from ATP to the substrate protein [1-3]. This phosphorylation induces conformational changes of the substrate protein leading to initiation of a number of cellular events including signal transduction [4,5]. The human PKC enzyme comprises of a number of isozymes and inappropriate activation of PKC has been linked to a variety of disorders [6,7]. The development of selective PKC inhibitors as novel therapeutics has therefore remained significant [8-14].

Balanol ((-)-1, Figure 1), a fungal metabolite [15] is known to inhibit a number of PKC isozymes at nanomolar concentrations [16], a finding that has motivated research related to the total[17-26] or fragment synthesis [27-47] of this important natural product. Based on the information $[48,49]$ that balanol binds to the ATP-docking site of protein kinase, all the three distinct domains present in the natural product such as the benzophenone core [50-52], the azepine core [53-59] and the<smiles>O=C(NC1CNCCCC1OC(=O)c1cc(O)c(C(=O)c2c(O)cccc2C(=O)O)c(O)c1)c1ccc(O)cc1</smiles>

Figure 1: Balanol (1) and ophiocordin (2). 
$p$-hydroxybenzamide $[60,61]$ unit have been targeted for analogue design in the quest for a more selective drug candidate over the last two decades. Although remarkable achievements have been made, the development of a unified synthetic strategy that would allow access to the natural product itself as well as some of its analogues remains important. A similar target is the closely related natural product ophiocordin (2). Herein, we describe a general approach to some of these targets.

\section{Results and Discussion}

The key feature of our retrosynthetic analysis (Figure 2) is the identification of the dehydro derivative of balanol 4 as the unified precursor of balanol (1) and an azepin ring-modified balanol 3. Derivative 4 could be obtained through esterification between the carboxylic acid $\mathbf{5}$ and the allylic alcohol $\mathbf{6}$.

We thus focused on the synthesis of the two key fragments 5 and 6 . The synthesis of the benzophenone unit has previously been achieved by several groups [27-30]. We adopted some of these methodologies with a number of modifications to prepare fragment 5 in its protected form 7 (Scheme 1). At first, the reaction of the known [17] bromo compound 8 with the known [27] aldehyde 9 in the presence of butyllithium effected a smooth conversion to the new benzylic alcohol 10. The latter was oxidized with tetrapropylammonium perruthenate to provide the benzophenone derivative $\mathbf{1 1}$ in good yield. Subsequent cleavage of the 1,3-dioxane unit followed by oxidation of the resulting aldehyde $\mathbf{1 2}$ furnished carboxylic acid $\mathbf{1 3}$ in 73\% overall yield over two steps. Concomitant removal of the phenolic MOM ether and the alcoholic TBDPS ether protecting groups in $\mathbf{1 3}$ under acidic conditions proceeded without significant loss of product to provide the dihydroxy acid $\mathbf{1 4}$ in good yield. Reaction of $\mathbf{1 4}$ with an excess of benzyl bromide in the presence of $\mathrm{K}_{2} \mathrm{CO}_{3}$ afforded simultaneous protection of the phenolic $\mathrm{OH}$ and the carboxylic acid functions leaving the primary alcohol function unprotected, as desired. Compound $\mathbf{1 5}$ was then converted following a literature procedure into the known [17] benzophenone 7 through two consecutive oxidations involving the aldehyde $\mathbf{1 6}$ as the intermediate. Taken as a hole the described synthesis of $\mathbf{7}$ from $\mathbf{8}$ and $\mathbf{9}$ proceeded in eight linear steps in an overall yield of $22 \%$.

The synthesis of the azepine unit [31-47] was achieved following our preliminary report [62]. Thus, reductive amination of Garner's aldehyde 17 (Scheme 2) with allylamine produced amine 18 which was $\mathrm{N}$-protected with $\mathrm{CbzCl}$ to obtain 19 in an overall yield of $89 \%$ over three steps. The oxazolidine ring in compound $\mathbf{1 9}$ was then cleaved under acidic conditions and the resulting primary alcohol $\mathbf{2 0}$ was oxidized carefully under modified Swern conditions [63] to provide the $\alpha$-chiral aldehyde 21 which was used directly in the next step. Addition of vinylmagnesium bromide to aldehyde $\mathbf{2 1}$ under optimized conditions gave a separable mixture of the allylic alcohols 22 and $\mathbf{2 3}$ in a combined yield of $64 \%$ over two steps. The undesired anti-isomer $\mathbf{2 3}$ could be effectively converted to the desired $s y n$-isomer 22 by a Mitsunobu-type inversion [64].

The major $s y n$-isomer $\mathbf{2 2}$ was then acetylated and the resulting diene 24 was subjected to ring-closing metathesis [65] in the presence of Grubbs' second generation catalyst, benzylidene[1,3-bis(2,4,6-trimethylphenyl)-2-imidazolidinylidene]dichloro-(tricyclohexylphosphine)ruthenium (25). Pleasingly, the desired cycloalkene $\mathbf{2 6}$ was obtained in a gratifying yield of $89 \%$. The sequential removal of the $O$-acetyl group leading to $\mathbf{2 7}$ followed by removal of the $N$-Boc group in the latter was executed under standard conditions to provide amine 28. This was then coupled with 4-benzyloxybenzoic acid using EDC as activating agent to obtain the corresponding amide<smiles>[X][C]1C([X])CNC[C@@H](NC(=O)c2ccc(O)cc2)[C@@H]1OC(=O)c1cc(O)c(C(=O)c2c(O)cccc2CO)c(O)c1</smiles>

1, $\mathrm{X}=\mathrm{H}$

$3, \mathrm{X}=\mathrm{OH}$<smiles>CO[C@H]1C=CCNC[C@@H]1NC(=O)c1ccc(O)cc1</smiles>

4<smiles>O=C(O)c1cc(O)c(C(=O)c2c(O)cccc2O)c(O)c1</smiles>

6

Figure 2: Strategic bond disconnections of balanol. 


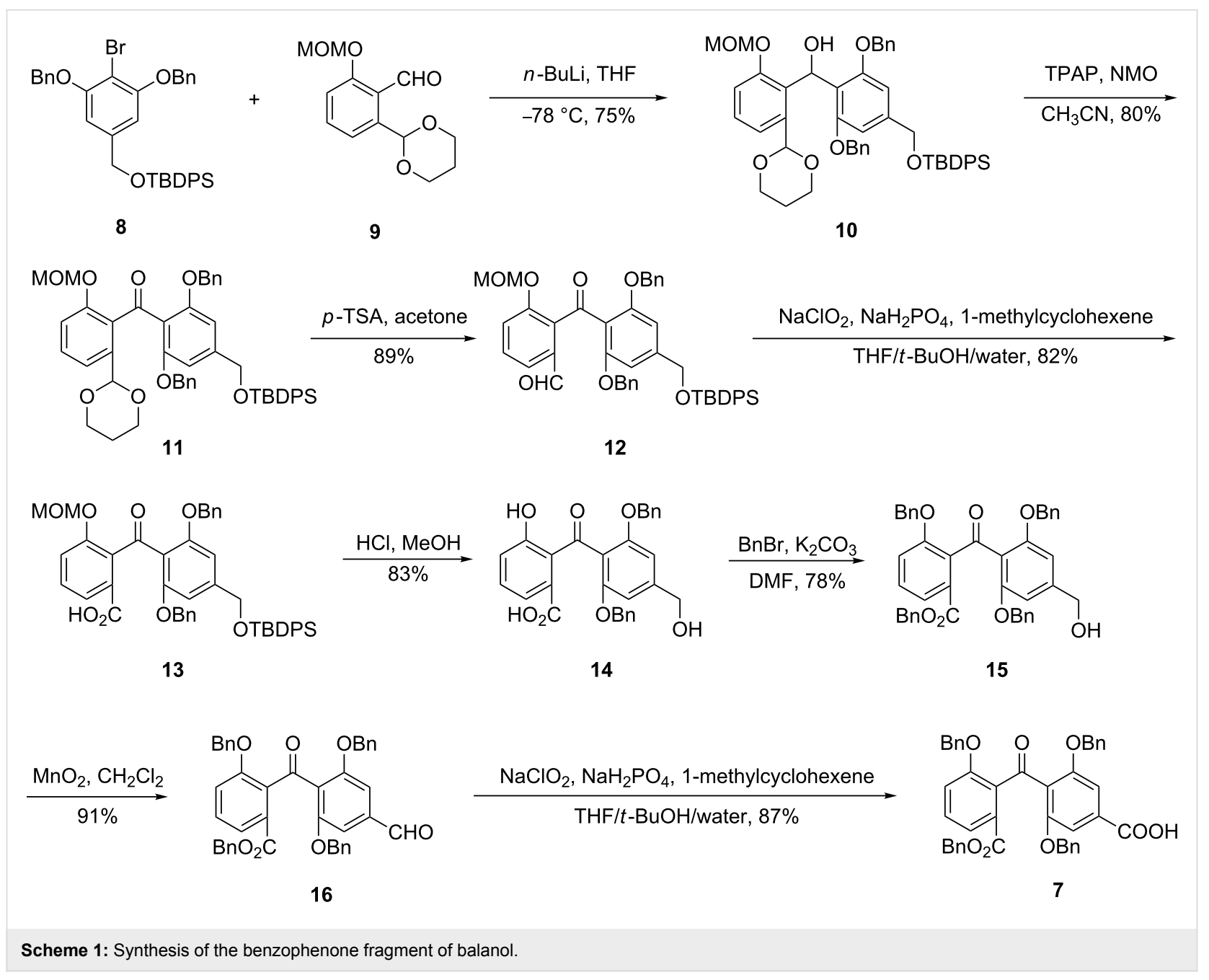

derivative $\mathbf{2 9}$ in an overall yield of $20 \%$ over eleven steps from 17. The stereochemical identity of this tetrahydroazepine derivative was confirmed by its selective conversion to the corresponding known azepane derivative $\mathbf{3 0}$ which displayed optical and ${ }^{13} \mathrm{C}$ NMR data nearly overlapping with those reported by Nicolaou et al [17].

With the two key fragments $\mathbf{2 9}$ and $\mathbf{7}$ in hand, we next focused on their convergent combination. The esterification of the allylic alcohol 29 with the acid 7 (Scheme 3) proceeded best in the presence of Mukaiyama's reagent [66], 2-chloro-1methylpyridinium iodide, to provide the ester $\mathbf{3 1}$ in $73 \%$ yield. Simultaneous hydrogenolytic removal of the $O$-benzyl groups and the $N$-Cbz group under reported conditions finally provided the natural (-)-balanol in a yield of $41 \%$. The product thus obtained displayed spectroscopic and optical data in close agreement to those reported for natural balanol [17].

We next focused our attention to demonstrate the utility of the intermediate coupled product $\mathbf{3 1}$ in a possible synthesis of an azepane ring-modified balanol derivative along the projected pathway. To this end, dihydroxylation of the adduct 31 was next attempted. Pleasingly, the dihydroxylation of $\mathbf{3 1}$ proceeded smoothly; however, unfortunately to provide an inseparable mixture of the two possible dihydroxylated isomers $\mathbf{3 2}$ in a combined yield of $68 \%$. The isomeric composition of $\mathbf{3 2}$ was determined to be $81: 19$ by HPLC.

\section{Conclusion}

In conclusion, we have developed a concise synthetic approach to the naturally occurring (-)-balanol (1) from easily available starting materials and reagents. Most of the synthetic steps proceeded in good to very good overall yield and stereocontrol. The developed synthesis may therefore be a complement to the existing literature. An attempted synthesis of an azepane ring-modified balanol derivative from a common precursor unfortunately was unsuccessful due to difficulty in separating stereoisomeric products. However, the intermediate $\mathbf{3 1}$ may prove to be useful in the synthesis of other analogues. 

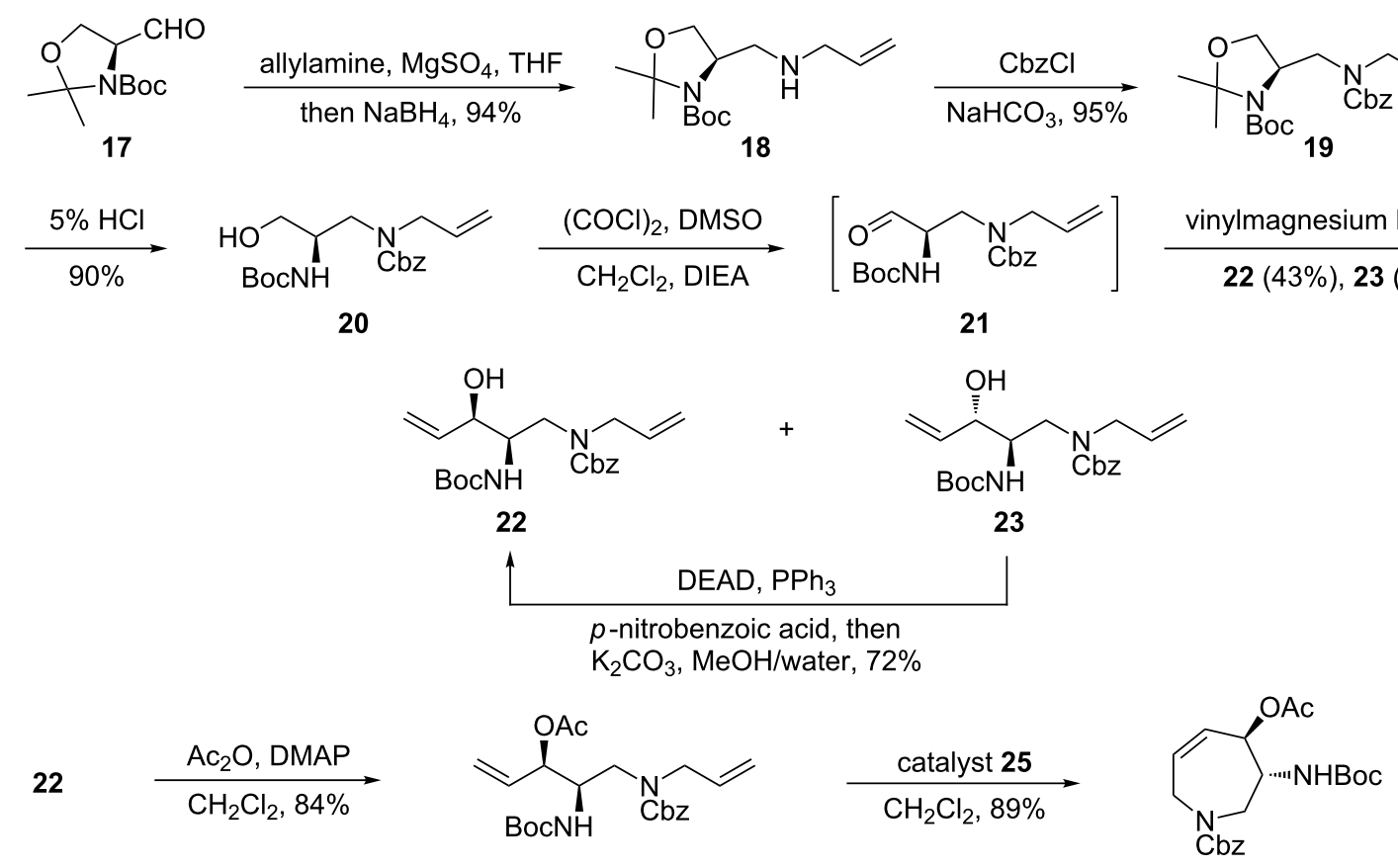

$\underset{\mathrm{MeOH}, 89 \%}{\stackrel{\mathrm{K}_{2} \mathrm{CO}_{3}}{\longrightarrow}} \stackrel{\mathrm{Cbz}_{\mathrm{Cb}}}{\stackrel{\mathrm{TFA} / \mathrm{CH}_{2} \mathrm{Cl}_{2}(1: 1)}{76 \%}}$

27<smiles>O=C(N[C@H]1CN(C(=O)OCc2ccccc2)CC=CC1O)c1ccc(OCc2ccccc2)cc1</smiles>

29

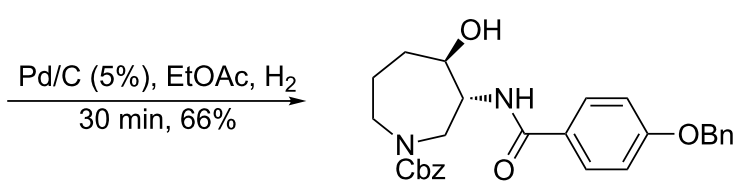

30

Scheme 2: Synthesis of the hexahydroazepine core of balanol.

$29+7 \stackrel{\text { 2-chloro-1-methylpyridinium iodide }}{\mathrm{Et}_{3} \mathrm{~N}, \mathrm{DMAP}, 73 \%}$<smiles>O=C(N[C@H]1CN(C(=O)O)C[C@@H](O)[C@H](O)[C@H]1OC(=O)c1ccccc1)c1ccc(OCc2ccccc2)c(Br)c1</smiles><smiles>O=C(O)c1cc(Br)c(C(=O)c2c(Br)cccc2OCc2ccccc2)c(OCc2ccccc2)c1</smiles><smiles>C1=C2CCCC2OCCO1</smiles>
$22(43 \%), 23(21 \%)$ 


\section{Supporting Information}

\section{Supporting Information File 1}

Experimental details and characterization data for the prepared compounds, copies of ${ }^{1} \mathrm{H}$ and ${ }^{13} \mathrm{C}$ NMR spectra of all new compounds, and data for the comparison of $\mathbf{3 0}$ and 1 with reported data.

[http://www.beilstein-journals.org/bjoc/content/ supplementary/1860-5397-9-327-S1.pdf]

\section{Acknowledgements}

We are thankful to DST, New Delhi for financial support (Grant No. SR/S1/OC-92/2012), and CSIR, New Delhi for fellowships (to T.S and R.M.). We are also thankful to the DST-PURSE program for partial support.

\section{References}

1. Nishizuka, Y. Nature 1984, 308, 693-698. doi:10.1038/308693a0

2. Nishizuka, Y. Science 1986, 233, 305-312. doi:10.1126/science. 3014651

3. Newton, A. C. J. Biol. Chem. 1995, 270, 28495-28498. doi:10.1074/jbc.270.48.28495

4. Nishizuka, Y. FASEB J. 1995, 9, 484-496.

5. Roffey, J.; Rosse, C.; Linch, M.; Hibbert, A.; McDonald, N. Q.; Parker, P. J. Curr. Opin. Cell Biol. 2009, 21, 268-279. doi:10.1016/j.ceb.2009.01.019

6. Wong, C. F.; Bairy, S. Curr. Pharm. Des. 2013, 19, 4739-4754. doi:10.2174/1381612811319260006

7. Mochly-Rosen, D.; Das, K.; Grimes, K. V. Nat. Rev. Drug Discovery 2012, 11, 937-957. doi:10.1038/nrd3871

8. Nishizuka, Y. Nature 2002, 334, 661-665. doi:10.1038/334661a0

9. Mellor, H.; Parker, P. J. Biochem. J. 1998, 332, 281-292.

10. Gomez, D. E.; Skilton, G.; Alonso, D. F.; Kazanietz, M. G. Oncol. Rep. 1999, 6, 1363-1370.

11. Lee, M. R.; Duan, W.; Tan, S.-L. Expert Opin. Ther. Targets 2008, 12, 535-552. doi:10.1517/14728222.12.5.535

12. Teicher, B. A. Clin. Cancer Res. 2006, 12, 5336-5345. doi:10.1158/1078-0432.CCR-06-0945

13. Hofmann, J. Curr. Cancer Drug Targets 2004, 4, 125-146. doi:10.2174/1568009043481579

14. Cohen, P. Nat. Rev. Drug Discovery 2002, 1, 309-315. doi: $10.1038 / \mathrm{nrd} 773$

15. Kulanthaivel, P.; Hallock, Y. F.; Boros, C.; Hamilton, S. M.; Janzen, W. P.; Ballas, L. M.; Loomis, C. R.; Jiang, J. B. J. Am. Chem. Soc. 1993, 115, 6452-6453. doi:10.1021/ja00067a087

16. Ohshima, S.; Yanagisawa, M.; Katoh, A.; Fujii, T.; Sano, T.; Matsukuma, S.; Furumai, T.; Fujiu, M.; Watanabe, K.; Yokose, K.; Arisawa, M.; Okuda, T. J. Antibiot. 1994, 47, 639-647. doi:10.7164/antibiotics.47.639

17. Nicolaou, K. C.; Bunnage, M. E.; Koide, K. J. Am. Chem. Soc. 1994, 116, 8402-8403. doi:10.1021/ja00097a072

18. Lampe, J. W.; Hughes, P. F.; Biggers, C. K.; Smith, S. H.; Hu, H. J. Org. Chem. 1994, 59, 5147-5148. doi:10.1021/jo00097a014

19. Lampe, J. W.; Hughes, P. F.; Biggers, C. K.; Smith, S. H.; Hu, H. J. Org. Chem. 1996, 61, 4572-4581. doi:10.1021/jo952280k
20. Adams, C. P.; Fairway, S. M.; Hardy, C. J.; Hibbs, D. E.; Hursthouse, M. B.; Morley, A. D.; Sharp, B. W.; Vicker, N.; Warner, I. J. Chem. Soc., Perkin Trans. 1 1995, 2355-2362. doi:10.1039/P19950002355

21. Tanner, D.; Almario, A.; Högberg, T. Tetrahedron 1995, 51 , 6061-6070. doi:10.1016/0040-4020(95)00264-9

22. Tanner, D.; Tedenborg, L.; Almario, A.; Pettersson, I.; Csöregh, I.; Kelly, N. M.; Andersson, P. G.; Högberg, T. Tetrahedron 1997, 53, 4857-4868. doi:10.1016/S0040-4020(97)00167-1

23. Barbier, P.; Stadlwieser, J. Chimia 1996, 50, 530-532.

24. Miyabe, H.; Torieda, M.; Kiguchi, T.; Naito, T. Synlett 1997, 580-582. doi:10.1055/s-1997-3236

25. Miyabe, H.; Torieda, M.; Inoue, K.; Tajiri, K.; Kiguchi, T.; Naito, T. J. Org. Chem. 1998, 63, 4397-4407. doi:10.1021/j0980208r

26. Srivastava, A. K.; Panda, G. Chem.-Eur. J. 2008, 14, 4675-4688. doi:10.1002/chem.200701991

27. Hollinshead, S. P.; Nichols, J. B.; Wilson, J. W. J. Org. Chem. 1994, 59, 6703-6709. doi:10.1021/jo00101a032

28. Storm, J. P.; Andersson, C.-M. Org. Lett. 1999, 1, 1451-1453. doi:10.1021/ol9910060

29. Laursen, B.; Denieul, M.-P.; Skrydstrup, T. Tetrahedron 2002, 58, 2231-2238. doi:10.1016/S0040-4020(02)00096-0

30. Patil, M. L.; Deshpande, V. H.; Ramlingam, S.; Borate, H. B. Tetrahedron 2004, 60, 1869-1873. doi:10.1016/j.tet.2003.12.029

31. Oh, H.-S.; Kang, H.-Y. Bull. Korean Chem. Soc. 2012, 33, 3895-3898. doi:10.5012/bkcs.2012.33.11.3895

32. Louvel, J.; Chemla, F.; Demont, E.; Ferreira, F.; Pérez-Luna, A.; Voituriez, A. Adv. Synth. Catal. 2011, 353, 2137-2151. doi:10.1002/adsc.201100333

33. Hudlický, T. Pure Appl. Chem. 2010, 82, 1785-1796. doi:10.1351/PAC-CON-09-10-07

34. Gilmet, J.; Sullivan, B.; Hudlicky, T. Tetrahedron 2009, 65, 212-220. doi:10.1016/j.tet.2008.10.070

35. Trost, B. M.; Fandrick, D. R.; Brodmann, T.; Stiles, T. Angew. Chem., Int. Ed. 2007, 46, 6123-6125. doi:10.1002/anie.200700835

36. Unthank, M. G.; Hussain, N.; Aggarwal, V. K. Angew. Chem., Int. Ed. 2006, 45, 7066-7069. doi:10.1002/anie.200602782

37. Raghavan, S.; Kumar, C. N. Tetrahedron Lett. 2006, 47, 1585-1588. doi:10.1016/j.tetlet.2005.12.123

38. Yadav, J. S.; Srinivas, C. Tetrahedron 2003, 59, 10325-10329 doi:10.1016/j.tet.2003.09.089

39. Fürstner, A.; Thiel, O. R. J. Org. Chem. 2000, 65, 1738-1742. doi:10.1021/jo991611g

40. Masse, C. E.; Morgan, A. J.; Panek, J. S. Org. Lett. 2000, 2, 2571-2573. doi:10.1021/ol0061034

41. Riber, D.; Hazell, R.; Skrydstrup, T. J. Org. Chem. 2000, 65, 5382-5390. doi:10.1021/jo000538n

42. Phansavath, P.; de Paule, S. D.; Ratovelomanana-Vidal, V.; Genêt, J.-P. Eur. J. Org. Chem. 2000, 3903-3907. doi:10.1002/1099-0690(200012)2000:23<3903::AID-EJOC3903>3.0.C $\mathrm{O} ; 2-\mathrm{Q}$

43. Cook, G. R.; Shanker, P. S.; Peterson, S. L. Org. Lett. 1999, 1, 615-618. doi:10.1021/ol990705+

44. Wu, M. H.; Jacobsen, E. N. Tetrahedron Lett. 1997, 38, 1693-1696. doi:10.1016/S0040-4039(97)00192-5

45. Albertini, E.; Barco, A.; Benetti, S.; Risi, C. D.; Pollini, G. P.; Zanirato, V. Tetrahedron 1997, 53, 17177-17194. doi:10.1016/S0040-4020(97)10139-9 
46. Naito, T.; Torieda, M.; Tajiri, K.; Ninomiya, I.; Kiguchi, T. Chem. Pharm. Bull. 1996, 44, 624-626. doi:10.1248/cpb.44.624

47. Hu, H.; Jagdmann, G. E., Jr.; Hughes, P. F.; Nichols, J. B. Tetrahedron Lett. 1995, 36, 3659-3662. doi:10.1016/0040-4039(95)00623-K

48. Narayana, N.; Diller, T. C.; Koide, K.; Bunnage, M. E.; Nicolaou, K. C.; Brunton, L. L.; Xuong, N.-H.; Ten Eyck, L. F.; Taylor, S. S. Biochemistry 1999, 38, 2367-2376. doi:10.1021/bi9820659

49. Koide, K.; Bunnage, M. E.; Gomez Paloma, L.; Kanter, J. P.; Taylor, S. S.; Brunton, L. L.; Nicolaou, K. C. Chem. Biol. 1995, 2, 601-608. doi:10.1016/1074-5521(95)90124-8

50. Nicolaou, K. C.; Koide, K.; Bunnage, M. E. Chem.-Eur. J. 1995, 1, 454-466. doi:10.1002/chem.19950010711

51. Lampe, J. W.; Biggers, C. K.; Defauw, J. M.; Foglesong, R. J.; Hall, S. E.; Heerding, J. M.; Hollinshead, S. P.; Hu, H.; Hughes, P. F.; Jagdmann, G. E., Jr.; Johnson, M. G.; Lai, Y.-S.; Lowden, C. T.; Lynch, M. P.; Mendoza, J. S.; Murphy, M. M.; Wilson, J. W.; Ballas, L. M.; Carter, K.; Darges, J. W.; Davis, J. E.; Hubbard, F. R.; Stamper, M. L. J. Med. Chem. 2002, 45, 2624-2643. doi:10.1021/jm020018f

52. Breitenlechner, C. B.; Wegge, T.; Berillon, L.; Graul, K.; Marzenell, K.; Friebe, W.-G.; Thomas, U.; Schumacher, R.; Huber, R.; Engh, R. A.; Masjost, B. J. Med. Chem. 2004, 47, 1375-1390. doi:10.1021/jm0310479

53. Lai, Y.-S.; Stamper, M. Bioorg. Med. Chem. Lett. 1995, 5, 2147-2150. doi:10.1016/0960-894X(95)00364-Y

54. Lai, Y.-S.; Menaldino, D. S.; Nichols, J. B.; Jagdmann, G. E., Jr..; Mylott, F.; Gillespie, J.; Hall, S. E. Bioorg. Med. Chem. Lett. 1995, 5, 2151-2154. doi:10.1016/0960-894X(95)00365-Z

55. Mendoza, J. S.; Jagdmann, G. E., Jr.; Gosnell, P. A. Bioorg. Med. Chem. Lett. 1995, 5, 2211-2216. doi:10.1016/0960-894X(95)00382-4

56. Crane, H. M.; Menaldino, D. S.; Jagdmann, G. E., Jr.; Darges, J. W.; Buben, J. A. Bioorg. Med. Chem. Lett. 1995, 5, 2133-2138. doi:10.1016/0960-894X(95)00361-V

57. Defauw, J. M.; Murphy, M. M.; Jagdmann, G. E., Jr.; Hu, H.; Lampe, J. W.; Hollinshead, S. P.; Mitchell, T. J.; Crane, H. M.; Heerding, J. M.; Mendoza, J. S.; Davis, J. E.; Darges, J. W.; Hubbard, F. R.; Hall, S. E. J. Med. Chem. 1996, 39, 5215-5227. doi:10.1021/jm960581w

58. Hu, H.; Hollinshead, S. P.; Hall, S. E.; Kalter, K.; Ballas, L. M. Bioorg. Med. Chem. Lett. 1996, 6, 973-978. doi:10.1016/0960-894X(96)00151-5

59. Lai, Y.-S.; Mendoza, J. S.; Jagdmann, G. E., Jr.; Menaldino, D. S.; Biggers, C. K.; Heerding, J. M.; Wilson, J. W.; Hall, S. E.; Jiang, J. B.; Janzen, W. P.; Ballas, L. M. J. Med. Chem. 1997, 40, 226-235. doi:10.1021/jm960497g

60. Jagdmann, G. E., Jr.; Dafauw, J. M.; Lampe, J. W.; Darges, J. W.; Kalter, K. Bioorg. Med. Chem. Lett. 1996, 6, 1759-1764. doi:10.1016/0960-894X(96)00311-3

61. Hu, H.; Mendoza, J. S.; Lowden, C. T.; Ballas, L. M.; Janzen, W. P. Bioorg. Med. Chem. 1997, 5, 1873-1882. doi:10.1016/S0968-0896(97)00125-9

62. Roy, S. P.; Chattopadhyay, S. K. Tetrahedron Lett. 2008, 49, 5498-5501. doi:10.1016/j.tetlet.2008.07.031

63. Mancuso, A. J.; Swern, D. Synthesis 1981, 165-185. doi:10.1055/s-1981-29377

64. Grubbs, R. H.; Schrock, R. R.; Fürstner, A., Eds. Olefin Metathesis. Adv. Synth. Catal. 2007, 349, 1-265. doi:10.1002/adsc.200790000
65. Mukaiyama, T. Angew. Chem., Int. Ed. Engl. 1979, 18, 707-721. doi:10.1002/anie.197907073

66. Mitsunobu, O. Synthesis 1981, 1-28. doi:10.1055/s-1981-29317

\section{License and Terms}

This is an Open Access article under the terms of the Creative Commons Attribution License (http://creativecommons.org/licenses/by/2.0), which permits unrestricted use, distribution, and reproduction in any medium, provided the original work is properly cited.

The license is subject to the Beilstein Journal of Organic Chemistry terms and conditions:

(http://www.beilstein-journals.org/bjoc)

The definitive version of this article is the electronic one which can be found at: doi:10.3762/bjoc. 9.327 